\title{
The Effect of Apolipoprotein E Polymorphism on Lipid Levels in Korean Adults
}

The aim of this study was to determine the effects of polymorphisms in the apolipoprotein $\mathrm{E}$ gene $(A P O E)$ on lipid levels in Korean adults and to investigate the interactions between these polymorphisms and environmental factors in determining lipid levels. We performed a cross-sectional study of 1,900 subjects (668 men and 1,232 women; 45-74 yr old) in Namwon, Korea, in 2004. APOE polymorphisms were determined by polymerase chain reaction and restriction enzyme analysis. Carriers of the $A P O E * E 2$ (E2) allele had significantly lower total cholesterol and low-density lipoprotein cholesterol (LDL-C) concentrations than did carriers of the $A P O E * E 3(E 3)$ or $A P O E * E 4(E 4)$ alleles, regardless of gender. The $A P O E$ allele type had significant effect on high-density lipoprotein cholesterol (HDL-C) and triglyceride levels in women, but not in men. The effect of APOE allele type on HDL-C levels was modified by age in women. In addition, in men, the effect of $A P O E$ allele type on triglyceride levels was modified by smoking. These findings highlight the important effect of gene-environment interactions on lipid levels.

Key Words : Apolipoproteins E; Polymorphism, Genetic; Lipids; Gene-Environment Interaction

\author{
Min-Ho Shin, Hee Nam Kim*, \\ Lian-Hua Cui', Sun-Seog Kweon, \\ Kyeong-Soo Park, Heon Heo, \\ Hae-Sung Nam, Seul-Ki Jeong ${ }^{\S}$, \\ Eun-Kyung Chung", Jin-Su Choi
}

Department of Preventive Medicine, Seonam University College of Medicine, Namwon, ${ }^{*}$ Genomic Research

Center of Hematopoietic Diseases, Chonnam National University Hospital, Gwangju, Korea; Department of Preventive Medicine ${ }^{\dagger}$, Yanbian University Medical College, Yanbian, China; Department of Preventive Medicinet, Chungnam University College of Medicine, Daejeon; Department of Neurology ${ }^{\text {s. " }}$, Chonbuk National University Hospital, Jeonju; 'Department of Preventive Medicine, Chonnam National University College of Medicine, "Chonnam National University Research Center of Medical Sciences, Gwangju, Korea

Received : 8 November 2004 Accepted : 28 December 2004

Address for correspondence

Eun-Kyung Chung, M.D.

Department of Preventive Medicine, Chonnam National University College of Medicine, Chonnam National University Research Center of Medical Sciences, 5 Hak 1-dong, Dong-gu, Gwangju 501-746, Korea Tel : +82.62-220-4175, Fax : +82.62-233-0305

E-mail : sehyukmom@hanmail.net

\section{INTRODUCTION}

Apolipoprotein E (apoE) is a plasma protein that serves as a ligand for low density lipoprotein receptors and, through its interaction with these receptors, participates in the transport of cholesterol and other lipids among the various cells in the body (1). The APOE gene is located at chromosome $19 \mathrm{q} 13.2$ and has three common variants $(A P O E * E 2, A P O E *$ $E 3$, and $A P O E * E 4 ; E 2, E 3$, and $E 4$, respectively) and six different genotypes $(E 2 / E 2, E 2 / E 3, E 2 / E 4, E 3 / E 3, E 3 / E 4$, and E4/E4) $(2,3)$. Allelic variation of APOE has been shown to influence the levels of serum lipids. Previous studies have consistently shown that total cholesterol (TC) and low-density lipoprotein cholesterol (LDL-C) are highest in $E 4$ carriers, intermediate in $E 3$ carriers, and lowest in $E 2$ carriers $(4,5)$; however, some reports have shown no effect of $A P O E$ genotype on LDL-C levels $(6,7)$. In contrast, the associations between $A P O E$ genotype and triglyceride and high density lipo- protein cholesterol (HDL-C) levels are less clear among different populations $(5,8)$. Moreover, the association between APOE genotype and lipid levels may be modulated by factors such as age, sex, ethnicity, diet, smoking behavior, alcohol consumption, and the presence of diabetes mellitus $(3,9,10)$. The aim of this study was to evaluate the association between APOE polymorphisms and lipid levels in a Korean population, and to explore the gene-environment interactions in this population.

\section{MATERIALS AND METHODS}

\section{Populations and study design}

Potential participants for this study were recruited from residents of five administrative regions (three Myuns and two Dongs) of Namwon in the Jeonbuk Province of Korea. We 
used national resident registration lists to identify potential participants. The study area contained an estimated 4,697 people (2,142 men and 2,555 women) who were between 45 to $74 \mathrm{yr}$ old in 2004 , the age limits for eligibility for the study. Among these 4,697 eligible subjects, 1,964 (41.8\%) underwent a clinical examination following an interview. The participants included 694 men and 1,270 women, and the mean ( \pm standard deviation) ages of the men and women were 61.9 $( \pm 6.7)$ and $61.1( \pm 7.1) \mathrm{yr}$, respectively. The response rate of women (48.4\%) was significantly higher than men $(33.5 \%)$, but the difference of mean ages between the respondent and non-respondent was not significant in both sexes. Subjects with missing lipid measurements or lifestyle data, or with triglyceride levels $\geq 800 \mathrm{mg} / \mathrm{dL}$ (11) were excluded from the study. The $A P O E$ genotypes were categorized into three groups: $A P O E * E 2$ (E2/E2 and E2/E3), APOE*E3 (E3/E3), and $A P O E * E 4$ (E3/E4 and E4/E4). Subjects with the E2/E4 genotype ( 4 men and 12 women) were excluded because of the expected opposing biological effects of the E2 and E4 alleles. Ultimately, 1,900 subjects (668 men and 1,232 women) formed the final study sample for the principal analysis. All participants provided informed consent, and the study was conducted in accordance with the approval of the institutional research ethics committee.

\section{Anthropometric measurements}

Anthropometric measurements of subjects wearing light clothing and no shoes were conducted by experienced research staff. Height was measured to the nearest $0.1 \mathrm{~cm}$, and weight was measured in the upright position to the nearest $0.1 \mathrm{~kg}$. The body mass index (BMI) was calculated as weight divided by height squared $\left(\mathrm{kg} / \mathrm{m}^{2}\right)$. Waist circumference was measured to the nearest $0.1 \mathrm{~cm}$ at the end of a normal expiration by measuring from the narrowest point between the lower border of the rib cage and the iliac crest.

\section{Lipid measurements}

All the participants underwent at least $10 \mathrm{hr}$ of overnight fasting before blood samples were obtained from an antecubital vein. Serum was separated on-site and was stored at $-70^{\circ} \mathrm{C}$ until analyzed. The concentrations of TC, HDL-C, and triglycerides were measured by an automatic analyzer (AU5400, Olympus, Japan), and LDL-C was estimated using the formula of Friedewald et al. (12). The fasting blood glucose level was measured using an enzymatic method.

\section{Measurement of environmental factors}

The questionnaire administered to all participants was designed to determine the history of type 2 diabetes mellitus and lifestyle factors such as alcohol consumption, smoking, and exercise habits. The subjects were categorized accord- ing to their smoking habits as current smokers, ex-smokers, and non-smokers. Type 2 diabetes mellitus was defined as a self-reported history (having been informed by a physician that diabetes was present) or as a fasting blood glucose level $\geq 126 \mathrm{mg} / \mathrm{dL}$.

\section{ApoE genotyping}

Genomic DNA was extracted from peripheral blood with a AccuPrep Genomic DNA Extraction Kit (Bioneer, Seoul, Korea) according to the manufacturer's protocol. APOE genotypes were determined as described by Hixson and Vernier, with slight modification (13). A 244-bp fragment of the $A P O E$ gene that spans the two polymorphic sites at codons 112 and 158 was amplified by polymerase chain reaction in a DNA thermal cycler (PTC-200; MJ Research, Inc, Watertown, MA, U.S.A.) using the primers 5 ' -ACA GAATTCGCCCCGGCCTGGTACAC-3 ${ }^{\prime}$ and 5' -TAAGCTTGGCACGGCTGTCCAAGGA-3' . The PCR amplifications were carried out in $10 \mu \mathrm{L}$ reaction volumes containing $0.25 \mathrm{mM}$ each dNTP, $1.5 \mathrm{mM}$ magnesium chloride, $0.75 \mathrm{U}$ of $\mathrm{f}-\mathrm{Taq}$ polymerase (Solgent, Seoul, Korea), 50 ng of genomic DNA, and $0.25 \mu \mathrm{M}$ of each of the primers. The PCR cycling conditions were $94^{\circ} \mathrm{C}$ for $5 \mathrm{~min}$, followed by 40 cycles of $94^{\circ} \mathrm{C}$ for $30 \mathrm{sec}, 65^{\circ} \mathrm{C}$ for $40 \mathrm{sec}$, and $72^{\circ} \mathrm{C}$ for $90 \mathrm{sec}$. A final extension phase of $5 \mathrm{~min}$ at $72^{\circ} \mathrm{C}$ was included at the end of the protocol. The PCR products were digested with 5 units of HhaI (TaKaRa Co. Ltd., Kyoto, Japan) and the fragments were separated by electrophoresis in $10 \%$ polyacrylamide gels. After electrophoresis, the gels were treated with ethidium bromide and the DNA fragments were visualized using ultraviolet illumination.

\section{Statistical analysis}

The differences between sexes were compared using Student's unpaired t-test for continuous data and the chi-square test for categorical data. Chi-square tests were conducted to compare the differences between the observed and expected frequencies, assuming Hardy-Weinberg equilibrium. Because the distributions of TC and HDL-C were skewed, the data were log-transformed prior to the analysis. Analysis of covariance (ANCOVA) was carried out using the general linear model procedure to exclude the effects of potential confounders (such as age, BMI, waist circumference, smoking, alcohol consumption, exercise, and diabetes mellitus) on lipid levels. The interaction effect between the APOE allele and demographic (sex and age) and environmental (smoking, alcohol consumption, exercise, and diabetes mellitus) factors was tested by introducing corresponding interaction terms in the ANCOVA model. Bonferroni tests were applied to correct for multiple comparisons. All analyses were performed using SPSS software version 11.0 (SPSS, Chicago, IL, U.S.A.). 


\section{RESULTS}

The descriptive characteristics of the study subjects are presented in Table 1 . The percentages of men who were current smokers, consumed alcohol, engaged in regular exercise, or

Table 1. Characteristics of the subjects $(n=1,900)$ by gender

\begin{tabular}{lccr}
\hline & $\begin{array}{c}\text { Men } \\
(\mathrm{n}=668)\end{array}$ & $\begin{array}{c}\text { Women } \\
(\mathrm{n}=1,232)\end{array}$ & \multicolumn{1}{c}{$p^{\star}$} \\
\hline Age $(\mathrm{yr})$ & $62.0 \pm 6.7$ & $61.2 \pm 7.1$ & 0.026 \\
Body mass index $\left(\mathrm{kg} / \mathrm{m}^{2}\right)$ & $23.8 \pm 2.9$ & $25.0 \pm 3.2$ & $<0.001$ \\
Waist circumference (cm) & $86.5 \pm 8.2$ & $87.8 \pm 8.8$ & 0.002 \\
Current smoker (\%) & 35.9 & 3.7 & $<0.001$ \\
Alcohol consumption (\%) & 62.9 & 26.2 & $<0.001$ \\
Regular exercise (\%) & 23.2 & 14.9 & $<0.001$ \\
Diabetes mellitus (\%) & 11.2 & 7.2 & 0.003 \\
Total cholesterol (mg/dL) & $190.5 \pm 39.3$ & $202.0 \pm 40.0$ & $<0.001$ \\
HDL cholesterol (mg/dL) & $46.0 \pm 13.0$ & $47.2 \pm 12.3$ & 0.050 \\
LDL cholesterol (mg/dL) & $109.1 \pm 35.7$ & $123.4 \pm 36.1$ & $<0.001$ \\
Triglycerides (mg/dL) & $177.0 \pm 113.5$ & $156.6 \pm 96.7$ & $<0.001$ \\
Apolipoprotein E genotypes ${ }^{\dagger}$ & & & \\
E2/E2 & 0.3 & 0.6 & \\
E2/E3 & 10.3 & 9.1 & \\
E2/E4 & 0.6 & 1.0 & 0.177 \\
E3/E3 & 75.3 & 72.9 & \\
E3/E4 & 12.6 & 15.3 & \\
E4/E4 & 0.7 & 0.9 & \\
\hline
\end{tabular}

Unless otherwise indicated, data are given as mean \pm standard deviation. HDL, high density lipoprotein; LDL, low density lipoprotein.

${ }^{*} p$ value, determined by t-test or chi-square test as appropriate, ${ }^{1}$ The sample consisted of 1,916 individuals (672 men, 1,244 women), including the 16 subjects with the APOE*E2/E4 genotype (4 men, 12 women). had diabetes mellitus were significantly higher than the corresponding percentages of women; however, women had significantly greater mean BMI and waist circumference figures than did men. The TC, LDL-C and HDL-C levels were significantly higher in women than in men, but the triglyceride levels were higher in men. The distribution of the APOE polymorphism in our study population was in Hardy-Weinberg equilibrium, and not significantly different in men and women. The genotype distribution was similar to those observed in other Korean studies $(14,15)$.

The relationship between the APOE genotypes and the serum lipid concentrations for male and female subjects is presented in Table 2 . The APOE allele type had a significant effect on TC and LDL-C levels in both men and women $(p<$ $0.05)$. E2 carriers had significantly lower TC and LDL-cholesterol concentrations than did E3 or E4 carriers. The APOE allele type had no significant effect on HDL-C levels in men, but there was a linear trend $(p=0.04)$. Among women, E2 or $E 3$ carriers had significantly higher HDL-C levels than did $E 4$ carriers. The APOE allele type had no significant effect on triglyceride levels in men; however, among women, E4 carriers had the highest and $E 3$ carriers had the lowest triglyceride levels. The associations between the APOE allele and lipid levels were not modified by sex (Table 2).

Table 3 shows the interaction effects between the APOE alleles and age and environmental factors in men and women. In women, the effect of the APOE allele type on HDL-C levels differed with age (Table 3). In women $<60 \mathrm{yr}$ old, E2 and E3 carriers had significantly higher HDL-C levels than did E4 carriers (Fig. 1). This pattern was similar to that in men

Table 2. Total cholesterol, low-density lipoprotein cholesterol, high-density lipoprotein cholesterol, and triglyceride levels according to APOE allele

\begin{tabular}{|c|c|c|c|c|c|c|}
\hline & $\begin{array}{c}E 2(E 2 / 2, E 2 / 3) \\
(\mathrm{n}=72 \text { Male, } 120 \text { Female })\end{array}$ & $\begin{array}{c}E 3(E 3 / 3) \\
(n=506 \text { Male, } 890 \text { Female })\end{array}$ & $\begin{array}{c}E 4(E 4 / 4, E 3 / 4) \\
(\mathrm{n}=90 \text { Male, } 222 \text { Female })\end{array}$ & $p^{*}$ & $p$ trend $^{\dagger}$ & $\begin{array}{c}\text { Interaction }^{\ddagger} \\
(\text { Sex } \times A P O E \\
\text { allele })\end{array}$ \\
\hline \multicolumn{7}{|c|}{ Total cholesterol } \\
\hline Male & $179.8(170.8-188.8)$ & $191.5(188.1-194.9)^{\S}$ & $193.2(185.1-201.2)$ & 0.045 & 0.041 & \\
\hline Female & $188.7(181.9-195.9)$ & $202.2(199.7-204.8)^{\S}$ & $208.1(203.0-213.3)^{\S}$ & $<0.001$ & $<0.001$ & 0.623 \\
\hline \multicolumn{7}{|c|}{ LDL cholesterol } \\
\hline Male & $92.9(84.9-100.9)$ & $110.5(107.6-113.5)^{\S}$ & $114.0(106.8-121.1)^{\S}$ & $<0.001$ & $<0.001$ & \\
\hline Female & $107.8(101.4-114.1)$ & $124.6(122.6-126.9)^{8}$ & $127.3(122.6-132.0)^{8}$ & $<0.001$ & $<0.001$ & 0.947 \\
\hline \multicolumn{7}{|c|}{ HDL cholesterol } \\
\hline Male & $46.0(43.4-48.8)$ & $44.5(43.5-45.5)$ & $42.4(40.2-44.7)$ & 0.117 & 0.040 & \\
\hline Female & $46.3(44.2-48.4)^{\pi}$ & $46.3(44.5-47.0)^{\pi}$ & $43.0(41.6-44.5)$ & 0.001 & 0.002 & 0.590 \\
\hline \multicolumn{7}{|c|}{ Triglyceride } \\
\hline Male & $163.2(143.9-185.0)$ & $146.1(139.3-153.1)$ & $152.6(136.3-170.7)$ & 0.241 & 0.530 & \\
\hline Female & $138.6(126.5-151.8)$ & $130.3(126.0-134.8)$ & $152.7(142.8-163.3)^{\|}$ & $<0.001$ & 0.009 & 0.093 \\
\hline
\end{tabular}

The mean lipid values were adjusted for age, BMI, waist circumference, smoking, alcohol, exercise, and diabetes mellitus, with 95\% confidence intervals given in parenthesis. Log-transformed triglyceride concentration data were used in the analysis and the mean obtained using the anti-log transformation. LDL, low density lipoprotein; HDL, high density lipoprotein.

* $p$ value by ANCOVA for global comparison between APOE alleles after adjusting for age, BMl, waist circumference, smoking, alcohol, exercise, and diabetes mellitus; ${ }^{\dagger} p$ value by ANCOVA for APOE alleles after adjusting for age, BMl, waist circumference, smoking, alcohol, exercise, and diabetes mellitus; ${ }^{\ddagger} p$ value by ANCOVA for interaction term (Sex $\times A P O E$ allele) after adjusting for age, BMI, waist circumference, smoking, alcohol, exercise, and diabetes mellitus; ${ }^{8}$ Significantly different from E2, $p<0.05$ (Bonferroni adjustment for multiple comparisons); "Significantly different from E3, $p<0.05$ (Bonferroni adjustment for multiple comparisons); "Significantly different from E4, $p<0.05$ (Bonferroni adjustment for multiple comparisons). 


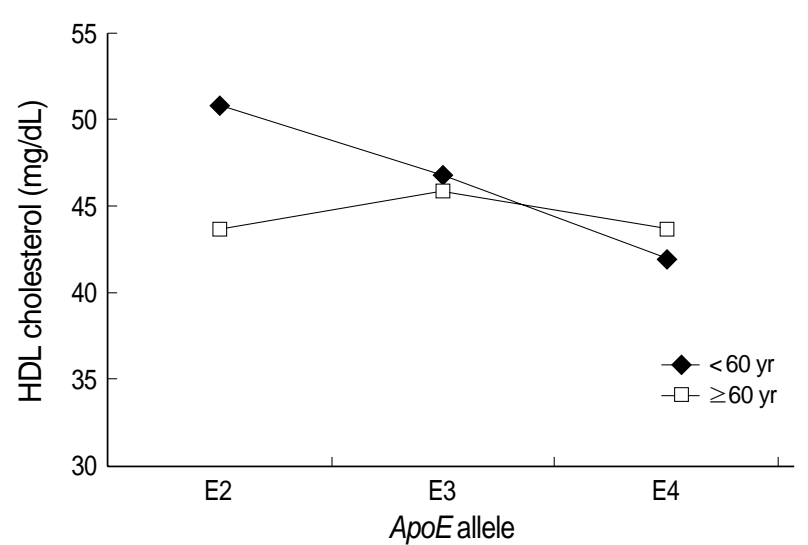

Fig. 1. Comparison of adjusted means of high density lipoprotein cholesterol (HDL-C) in females by APOE alleles and age subgroups. Mean HDL-C values are adjusted for age, BMI, waist circumference, smoking, alcohol, exercise, and diabetes mellitus. There was a significant interaction $(p<0.006)$ between age group and $A P O E$ allele type on HDL-C levels. The APOE allele type had a significant effect on HDL-C levels in women $<60$ yr old $(p<0.001)$ and in women $\geq 60 \mathrm{yr}$ old $(p=0.049$ ).

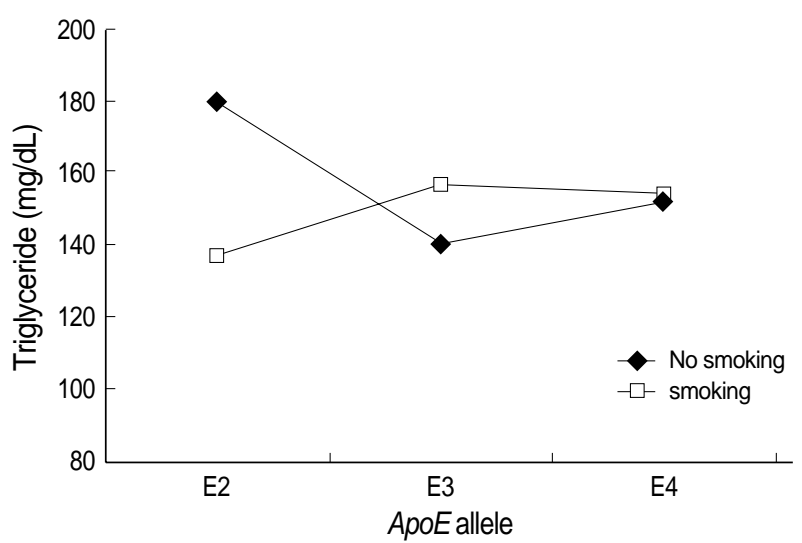

Fig. 2. Comparison of adjusted means of triglyceride levels in males by $A P O E$ alleles and smoking status. There was a significant effect of the interaction ( $p=0.022)$ between smoking status and APOE allele type on triglyceride levels. Mean triglyceride values are adjusted for age, BMI, waist circumference, alcohol, exercise, and diabetes mellitus. The APOE allele type had a significant effect on triglyceride levels in non-smokers ( $p=0.018)$, but no significant effect in smokers $(p=0.466)$.

Table 3. $p$-values for the effects of interaction between APOE alleles and age and environmental factors on lipid levels

\begin{tabular}{|c|c|c|c|c|c|c|c|c|}
\hline \multirow{2}{*}{ Interaction term } & \multicolumn{4}{|c|}{ Men $(n=668)$} & \multicolumn{4}{|c|}{ Women $(n=1,232)$} \\
\hline & TC & LDL-C & HDL-C & Triglyceride & TC & LDL-C & HDL-C & Triglyceride \\
\hline$A P O E$ alleles+age & 0.491 & 0.895 & 0.960 & 0.312 & 0.752 & 0.899 & 0.006 & 0.526 \\
\hline APOE alleles+smoking & 0.521 & 0.676 & 0.402 & 0.022 & 0.671 & 0.601 & 0.089 & 0.418 \\
\hline APOE alleles+alcohol intake & 0.476 & 0.893 & 0.680 & 0.592 & 0.496 & 0.635 & 0.727 & 0.342 \\
\hline APOE alleles+regular exercise & 0.192 & 0.713 & 0.412 & 0.245 & 0.687 & 0.807 & 0.776 & 0.662 \\
\hline APOE alleles+diabetes mellitus & 0.638 & 0.883 & 0.542 & 0.108 & 0.354 & 0.408 & 0.763 & 0.051 \\
\hline
\end{tabular}

TC, total cholesterol; LDL, low density lipoprotein; HDL, high density lipoprotein.

* $p$-value by ANCOVA for interaction term after adjusting for age, BMI, waist circumference, smoking, alcohol, exercise, and diabetes mellitus.

(Table 1). In women $\geq 60$ yr old, the $A P O E$ allele type still had a significant effect on HDL-C levels ( $p=0.049)$; $E 3$ carriers tended to have higher HDL-C levels than $E 2$ or $E 4$ carriers, but there was no significant association of the $A P O E$ allele type with HDL-C levels after Bonferroni adjustment for multiple comparisons (result not shown). In addition, in men, the effect of $A P O E$ allele type on triglyceride levels was modified by smoking (Fig. 2). Among non-smokers, the triglyceride levels were highest in E2 carriers and lowest in E3 carriers. Among current smokers, E3 carriers had the highest triglyceride levels and E2 carriers had the lowest triglyceride levels, but these differences were not significant.

\section{DISCUSSION}

In this large population-based study, we have shown that APOE polymorphisms had a significant effect on lipid levels and that gene-environment interactions significantly affected lipid levels in a Korean population. We found that E2 carriers had significantly lower TC and LDL-C levels than $\operatorname{did} E 3$ or
E4 carriers. This finding is consistent with previous studies, in which TC and LDL-C levels were the highest in E4 carriers, intermediate in $E 3$ carriers, and the lowest in $E 2$ carriers (5, $8,16,17)$. Our study also showed that $E 4$ carriers tended to have higher TC and LDL-C levels than E3 carriers, although this difference was small and not significant. However, a metaanalysis (17) showed that subjects with E3/E4 and E4/E4 genotypes had significantly higher TC levels than did subjects with the E3/E3 genotype. Frikke-Schmidt et al. (8) found that there was a stepwise increase in cholesterol and apoB levels, in both sexes, as a function of genotype (E2/E2, to E2/ $E 3$, to $E 2 / E 4$, to $E 3 / E 3$, to $E 3 / E 4$, to $E 4 / E 4$ ) and alleles (E2, to $E 3$, to $E 4)$. In a Singapore-based study (5), Tan et al. showed that $E 4$ carriers had higher TC and LDL-C levels than did E3 carriers among ethnic Chinese, but that there were no significant allelotype-related differences among ethnic Malays or Asian Indians of either sex, with the exception of the LDL$C$ levels in ethnic Malaysian women. Therefore, differences in TC and LDL-C levels between E3 carriers and E4 carriers may be variable across populations.

Our study also showed that the APOE allele type had a no 
significant effect on HDL-C levels in men, although a negative linear trend $(p=0.04)$ was present; however, in women, $E 2$ and $E 3$ carriers had significantly higher HDL-C levels than did $E 4$ carriers. Frikke-Schmidt et al. (8) showed that there was a stepwise decrease in HDL-C in women, but not in men, according to allelotype, as described above. In the Singapore study (5), Tan et al. showed that E2 carriers had the highest HDL-C levels and E4 carriers had the lowest HDL-C levels in the three ethnic groups studied. However, a metaanalysis (17) showed that there was no clear association between HDL-C levels and the APOE allele type, apart from the finding that HDL-C levels were significantly lower in $E 3 / E 4$ carriers than in $E 3 / E 3$ carriers.

We showed that female $E 4$ carriers and male E2 carriers had the highest triglyceride levels, and that $E 3$ carriers had the lowest triglyceride levels. This finding is in accordance with previous studies $(5,8,14,17,18)$. In a study of Korean men, Chun et al. (14) showed that APOE allele type was associated with the median serum triglyceride levels in descending order from $E 2 / E 3$, to $E 3 / E 4$, to $E 3 / E 3$. In the Singapore study (5), Tan et al. showed that $E 4$ carriers had higher triglyceride levels, as compared with $E 2$ and $E 3$ carriers, with the exception of ethnic Malaysian males, in whom triglyceride levels were the highest in E2 carriers. A meta-analysis (17) showed that triglycerides were higher in $E 2$ carriers and $E 4 /$ $E 3$ carriers than in E3/E3 carriers. Frikke-Schmidt et al. (8) showed that E2/E2 and E4/E4 carriers had the highest triglyceride levels, whereas E3/E3 carriers had the lowest triglyceride levels in both sexes. The higher levels of triglycerides observed in subjects carrying the $E 2$ allele can be explained by their slower plasma clearance of chylomicron and VLDL remnants $(17,19,20)$.

The association between $A P O E$ polymorphism and triglyceride and HDL-C levels are not entirely clear among different populations $(4,5,8,21)$. Gene-environment interaction may contribute to the discrepancies observed between studies. We found that the association between the APOE alleles and HDL-C levels was modified by age in women, and that the association between $A P O E$ alleles and triglyceride levels was modified by smoking status in men, and tended to be modified by the presence of diabetes mellitus in women $(p=$ 0.051). Previous studies have shown that HDL-C levels vary with physical activity, alcohol consumption, and diet $(9,22)$. Reznik et al. (23) showed that the association between APOE polymorphism and postprandial triglyceride clearance was modified by age, body weight, and fasting TG pool level. Some previous studies have also shown that the association between $A P O E$ polymorphism and lipid levels was modified by sex (24), but in our study, the associations between the $A P O E$ allele types and lipid levels were not modified by sex, with the exception of an association of borderline significance (0.093) for triglyceride levels. However, the underlying mechanisms responsible for the gene-environment interactions found in the present study are not clear. Such interactions imply that, at the molecular level, there is synergy between gene products, or between gene products and the by-products of environmental insults, resulting in greater than additive effects (25).

The present study has some limitations. First, the relatively small sample size in men prevented us from showing statistically significant associations, although the patterns for TC and HDL-C levels that were observed in men were similar to those observed in women. Second, the proportion of the study population with risk factors such as smoking in women, diabetes mellitus, and the APOE*E2 or E4 alleles was low, and this limited the power of this study to detect gene-environment interactions.

In summary, we have shown that $A P O E$ polymorphisms had a significant effect on lipid levels in Koreans, that the association between the APOE allele type and HDL-C levels was modified by age in women, and that the association between the APOE allele type and triglyceride levels was modified by smoking status in men. These findings highlight the important effect of gene-environment interactions on lipid levels.

\section{REFERENCES}

1. Mahley RW. Apolipoprotein E: cholesterol transport protein with expanding role in cell biology. Science 1988; 240: 622-30.

2. Utermann G, Hees M, Steinmetz A. Polymorphism of apolipoprotein E and occurrence of dysbetalipoproteinaemia in man. Nature 1977; 269: 604-7.

3. Davignon J, Gregg RE, Sing CF. Apolipoprotein E polymorphism and atherosclerosis. Arteriosclerosis 1988; 8: 1-21.

4. Corella D, Guillen M, Saiz C, Portoles O, Sabater A, Cortina S, Folch J, Gonzalez JI, Ordovas JM. Environmental factors modulate the effect of the APOE genetic polymorphism on plasma lipid concentrations: ecogenetic studies in a Mediterranean Spanish population. Metabolism 2001; 50: 936-44.

5. Tan CE, Tai ES, Tan CS, Chia KS, Lee J, Chew SK, Ordovas JM. APOE polymorphism and lipid profile in three ethnic groups in the Singapore population. Atherosclerosis 2003; 170: 253-60.

6. Deiana L, Pes GM, Carru C, Errigo A, Pettinato S, Carcassi C, Baggio G, Contu L. Lack of influence of apolipoprotein E4 on lipoprotein levels in the island population of Sardinia. Eur J Clin Invest 1998; 28: 290-4.

7. Aguilar CA, Talavera G, Ordovas JM, Barriguete JA, Guillen LE, Leco ME, Pedro-Botet J, Gonzalez-Barranco J, Gomez-Perez FJ, Rull JA. The apolipoprotein E4 allele is not associated with an abnormal lipid profile in a native American population following its traditional lifestyle. Atherosclerosis 1999; 142: 409-14.

8. Frikke-Schmidt R, Nordestgaard BG, Agerholm-Larsen B, Schnohr $\mathrm{P}$, Tybjaerg-Hansen A. Context-dependent and invariant associations between lipids, lipoproteins, and apolipoproteins and apolipoprotein E genotype. J Lipid Res 2000; 41: 1812-22.

9. Lussier-Cacan S, Bolduc A, Xhignesse M, Niyonsenga T, Sing CF. 
Impact of alcohol intake on measures of lipid metabolism depends on context defined by gender, body mass index, cigarette smoking, and apolipoprotein E genotype. Arterioscler Thromb Vasc Biol 2002; 22: 824-31.

10. Ordovas JM, Lopez-Miranda J, Mata P, Perez-Jimenez F, Lichtenstein $\mathrm{AH}$, Schaefer EJ. Gene-diet interaction in determining plasma lipid response to dietary intervention. Atherosclerosis 1995; 118 (Suppl): S11-27.

11. Tremblay AJ, Morrissette H, Gagne JM, Bergeron J, Gagne C, Couture P. Validation of the Friedewald formula for the determination of low-density lipoprotein cholesterol compared with beta-quantification in a large population. Clin Biochem 2004; 37: 785-90.

12. Friedewald WT, Levy RI, Fredrickson DS. Estimation of the concentration of low-density lipoprotein cholesterol in plasma, without use of the preparative ultracentrifuge. Clin Chem 1972; 18: 499-502.

13. Hixson JE, Vernier DT. Restriction isotyping of human apolipoprotein E by gene amplification and cleavage with HhaI. J Lipid Res 1990; 31: $545-8$

14. Chun S, Min WK, Kim JW, Park H, Jang S, Yang SE, Kim JQ. Apolipoprotein E polymorphism and serum lipoprotein (a) concentrations in a Korean male population. Ann Clin Biochem 2001; 38: 129-34.

15. Choi YH, Kim JH, Kim DK, Kim JW, Lee MS, Kim CH, Park SC. Distributions of ACE and APOE polymorphisms and their relations with dementia status in Korean centenarians. J Gerontol A Biol Sci Med Sci 2003; 58: 227-31.

16. Ehnholm C, Lukka M, Kuusi T, Nikkila E, Utermann G. Apolipoprotein E polymorphism in the Finnish population: gene frequencies and relation to lipoprotein concentrations. J Lipid Res 1986; 27: 227-35.

17. Dallongeville J, Lussier-Cacan S, Davignon J. Modulation of plasma triglyceride levels by apoE phenotype: a meta-analysis. J Lipid Res 1992; 33: 447-54.
18. Gomez-Coronado D, Alvarez JJ, Entrala A, Olmos JM, Herrera E, Lasuncion MA. Apolipoprotein E polymorphism in men and women from a Spanish population: allele frequencies and influence on plasma lipids and apolipoproteins. Atherosclerosis 1999; 147: 167-76.

19. Turner PR, Cortese C, Wootton R, Marenah C, Miller NE, Lewis B. Plasma apolipoprotein B metabolism in familial type III dysbetalipoproteinaemia. Eur J Clin Invest 1985; 15: 100-12.

20. Demant T, Bedford D, Packard CJ, Shepherd J. Influence of apolipoprotein E polymorphism on apolipoprotein B-100 metabolism in normolipemic subjects. J Clin Invest 1991; 88: 1490-501.

21. Muros M, Rodriguez-Ferrer C. Apolipoprotein E polymorphism influence on lipids, apolipoproteins and Lp (a) in a Spanish population underexpressing apoE4. Atherosclerosis 1996; 121: 13-21.

22. Nicklas BJ, Ferrell RE, Bunyard LB, Berman DM, Dennis KE, Goldberg AP. Effects of apolipoprotein E genotype on dietary-induced changes in high-density lipoprotein cholesterol in obese postmenopausal women. Metabolism 2002; 51: 853-8.

23. Reznik Y, Morello R, Pousse P, Mahoudeau J, Fradin S. The effect of age, body mass index, and fasting triglyceride level on postprandial lipemia is dependent on apolipoprotein E polymorphism in subjects with non-insulin-dependent diabetes mellitus. Metabolism 2002; 51: 1088-92.

24. Schaefer EJ, Lamon-Fava S, Johnson S, Ordovas JM, Schaefer MM, Castelli WP, Wilson PW. Effects of gender and menopausal status on the association of apolipoprotein E phenotype with plasma lipoprotein levels. Results from the Framingham Offspring Study. Arterioscler Thromb 1994; 14: 1105-13.

25. Talmud PJ, Humphries SE. Gene: environment interaction in lipid metabolism and effect on coronary heart disease risk. Curr Opin Lipidol 2002; 13: 149-54. 